\title{
Junctional Rhythm: A Rare Sign in Leptospirosis
}

\author{
Rachana Warrier, Santosh Kumar Singh¹, Sarvinder Singh, Ajai Kumar Tentu², Nidhi Singh, Chinmaya Dash, Vani Singh ${ }^{3}$ \\ Departments of Medicine and ${ }^{2}$ Pulmonary Medicine, Military Hospital, '1Department of Medicine, Medical Division, Military Hospital, ${ }^{3}$ Department of Radiation Oncology, \\ RIMS, Ranchi, Jharkhand, India
}

\section{Abstract}

Leptospirosis is an important re-emerging infectious disease. Leptospirosis has been estimated to affect tens of millions of humans annually with a case fatality rate ranging from $5 \%$ to $25 \%$; however, it is underreported due to the lack of clinical suspicion and barriers to diagnostic capacity. A 33-year-old healthy male presented with a history of fever of 2-day duration. His examination revealed icterus. His workup did not show any significant findings except for a mild transaminitis and a rise in serum creatinine by $0.5 \mathrm{mg} / \mathrm{dl}$. Later, the patient developed bradycardia. An ELISA test for Leptospira antibodies was positive. The patient recovered after a course of intravenous antibiotics. Leptospirosis should be considered early in the diagnosis of any patient who presents with acute, nonspecific febrile illness with multiorgan involvement. Our case had an atypical presentation with involvement of the cardiovascular system in the form of junctional rhythm.

Keywords: Atrioventricular block, junctional rhythm, leptospirosis, Weil's disease

\section{INTRODUCTION}

Leptospirosis is a zoonotic disease with worldwide distribution caused by Leptospira species, a pathogenic spirochete. The most important reservoirs are rodents. Urinary shedding of organisms from infected animals is the most significant source of Leptospira species. A majority of patients present with a subclinical disease in the form of a mild anicteric febrile illness. However, some may develop a severe form with multiorgan involvement, called Weil's disease. Weil's disease is characterized by multisystem dysfunction and can present with high fever, significant jaundice, renal failure, and hemorrhagic diathesis. It may also cause neurologic, pulmonary, or cardiovascular symptoms.

\section{Case Report}

A 33-year-old male presented to our hospital in the month of June with a history of fever, myalgia, severe bilateral calf pain, and headache of 2-day duration. He denied any history of recent travel.

His examination revealed a temperature of $101^{\circ} \mathrm{F}$, a pulse of 110 beats $/ \mathrm{min}$, and a blood pressure of $130 / 90 \mathrm{mmHg}$. The patient was alert and oriented. He was icteric. Systemic examination was otherwise essentially normal.

\begin{tabular}{|l|l|}
\hline \multicolumn{2}{|c|}{ Access this article online } \\
\hline Quick Response Code: & Website: \\
\hline & www.ijccm.org \\
\hline & \\
\hline
\end{tabular}

The initial laboratory results were notable for a creatinine of $1.5 \mathrm{mg} / \mathrm{dL}$, a total bilirubin of $4.9 \mathrm{mg} / \mathrm{dL}$, a direct bilirubin of $3.1 \mathrm{mg} / \mathrm{dL}$, aspartate aminotransferase of $96 \mathrm{U} / \mathrm{L}$, and alanine aminotransferase of $101 \mathrm{IU} / \mathrm{L}$. The urine analysis showed mild proteinuria. The electrocardiography (ECG) showed sinus tachycardia with 110 beats/min [Figure 1]. The patient was admitted for evaluation of his fever. He was given antipyretics and supportive management. Initial management was on the lines of acute hepatitis.

On the third day, the patient complained of restlessness. Examination revealed bradycardia with a pulse rate of 42 beats/min. His systemic examination was normal. The ECG now showed a junctional rhythm [Figures 2 and 3]. In view of his fever, transaminitis, deranged renal parameters, and cardiac involvement, he was evaluated for leptospirosis. His ELISA test for IgM Leptospira antibodies was positive. He was started on intravenous ceftriaxone.

His condition improved after the course of antibiotics, and his heart rate returned to a normal sinus rhythm with

Address for correspondence: Dr. Santosh Kumar Singh, Department of Medicine, Medical Division, Military Hospital, Namkum, Ranchi - 834 010, Jharkhand, India. E-mail: sksingh77@rediffmail.com

This is an open access journal, and articles are distributed under the terms of the Creative Commons Attribution-NonCommercial-ShareAlike 4.0 License, which allows others to remix, tweak, and build upon the work non-commercially, as long as appropriate credit is given and the new creations are licensed under the identical terms.

For reprints contact: reprints@medknow.com

How to cite this article: Warrier R, Singh SK, Singh S, Tentu AK, Singh N, Dash C, et al. Junctional rhythm: A rare sign in leptospirosis. Indian J Crit Care Med 2018;22:889-91. 


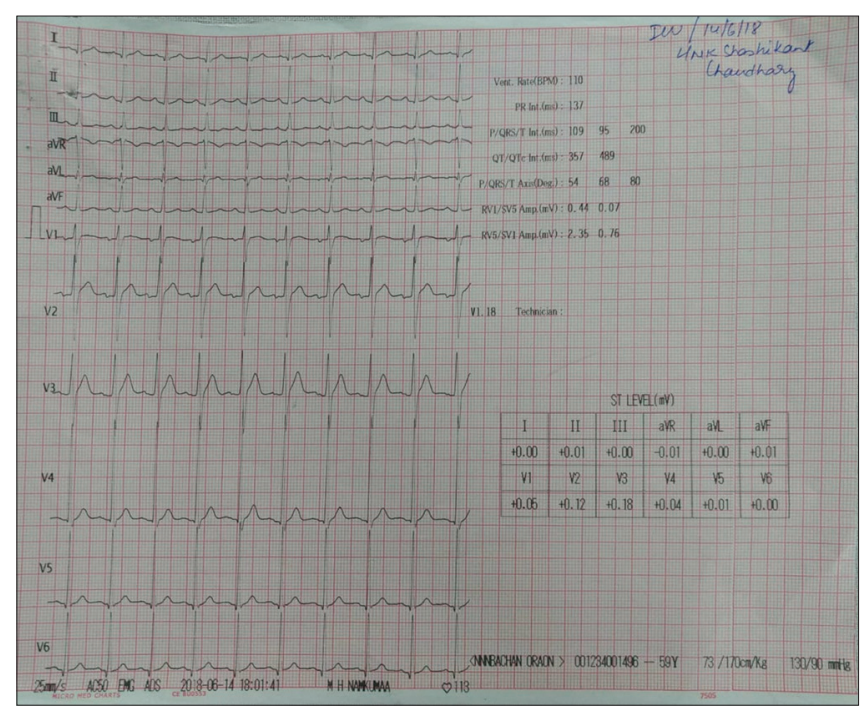

Figure 1: Electrocardiography showing sinus tachycardia

70 beats/min. We performed a Holter report, a two-dimensional echocardiography, and a myocardial stress test to rule out any structural cardiac abnormality. All of these examinations were normal.

\section{Discussion}

Leptospirosis is a zoonotic disease caused by infection with Leptospira interrogans, a pathogenic spirochete. It has over 200 different serovars, where some, such as Leptospira icterohaemorrhagica, are associated with various sinister complications and fatalities. The disease has been recognized in India since 1931 ${ }^{[1]}$ The incidence of leptospirosis as per the WHO is between 0.01 and 1 cases $/ 100,000$ in temperate, nonendemic areas and between 10 and 100 cases $/ 100,000$ in humid, tropical, endemic areas. ${ }^{[2]}$ In India, it is more endemic in the south of India, and only a few cases have been reported from the North. The organism infects a variety of animals, mostly rodents. Human cases arise from incidental infection usually through skin or mucous membranes after exposure to water or soil contaminated with the urine of an infected animal.

The incubation period is usually $1-2$ weeks but may range from 1 to 30 days. The natural course of leptospirosis has two distinct clinical phases, namely the leptospiremic phase and the immune phase. Complications occur in the second immune phase of the disease. Majority of the cases are relatively mild and present with a sudden onset of fever, headache, nausea, vomiting, abdominal pain, conjunctival suffusion, and myalgia. The complications of leptospirosis include renal failure, hepatitis, thrombocytopenia, pulmonary hemorrhage, acute respiratory distress syndrome, myocarditis, neuroleptospirosis, ocular complications, meningitis, hypotension, and hypokalemic paralysis. The immune phase of the disease develops through a combination of host factors and serovar type.

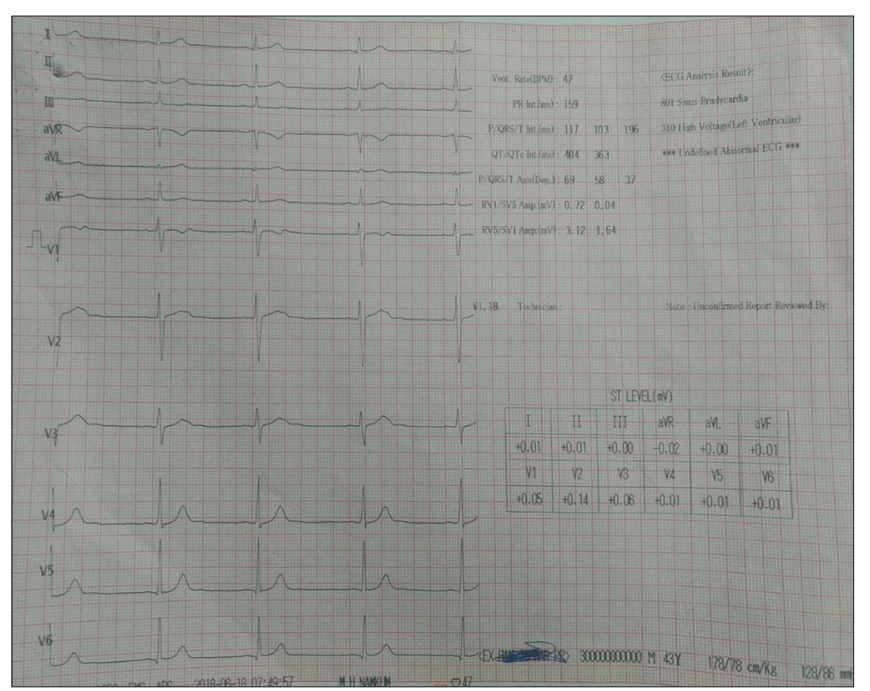

Figure 2: Electrocardiography showing junctional rhythm

\section{Cardiac involvement in leptospirosis}

A glycoprotein fraction of the leptospiral cell wall has been incriminated in the pathogenesis of cardiac rhythm disturbances. It is postulated that this protein inhibits the Na-K ATPase and causes arrhythmia. In one of the studies, analysis of 50 patients with leptospirosis had shown involvement of the cardiovascular system in approximately $70 \%$ patients, and the most common arrhythmia was atrial fibrillation. ${ }^{[3]} \mathrm{A}$ variety of electrocardiographic changes has been reported in leptospirosis. ${ }^{[4]}$ Atrial fibrillation, atrioventricular (AV) conduction blocks and nonspecific ventricular repolarization abnormalities are the most common. A study done by Trivedi et al. in South Gujarat on 25 seropositive patients of leptospirosis showed cardiovascular involvement in $14(56 \%)$ patients. Electrocardiographic changes were seen in $13(52 \%)$ patients. The most common arrhythmia seen in their study was first-degree AV block in 11 (44\%) patients, which, however, is seen rather frequently in young, healthy males as well..$^{[5]}$ Any echocardiographic evidence of myocardial dysfunction has not been adequately demonstrated. Histopathological changes of the myocardium may be found, in the form of myocardial inflammation and vasculitis. Cardiac involvement, demonstrated electrocardiographically or clinically, tends to predict poor outcome, and no specific therapies are available to prevent or treat cardiac involvement in leptospirosis. ${ }^{[6]}$

In the case of our patient, the diagnosis of leptospirosis was not considered in the initial phase. He was being managed on supportive measures as there was no risk factor. He did not travel anywhere, and Jharkhand is not endemic for leptospirosis. Only four cases were reported in the year 2015. However, he met with the Faine's criteria. Furthermore, junctional rhythm as a complication of leptospirosis has not been shown in previous studies. Our patient had an atypical presentation with cardiovascular manifestation in the form of junctional rhythm. After the diagnosis of leptospirosis, he 


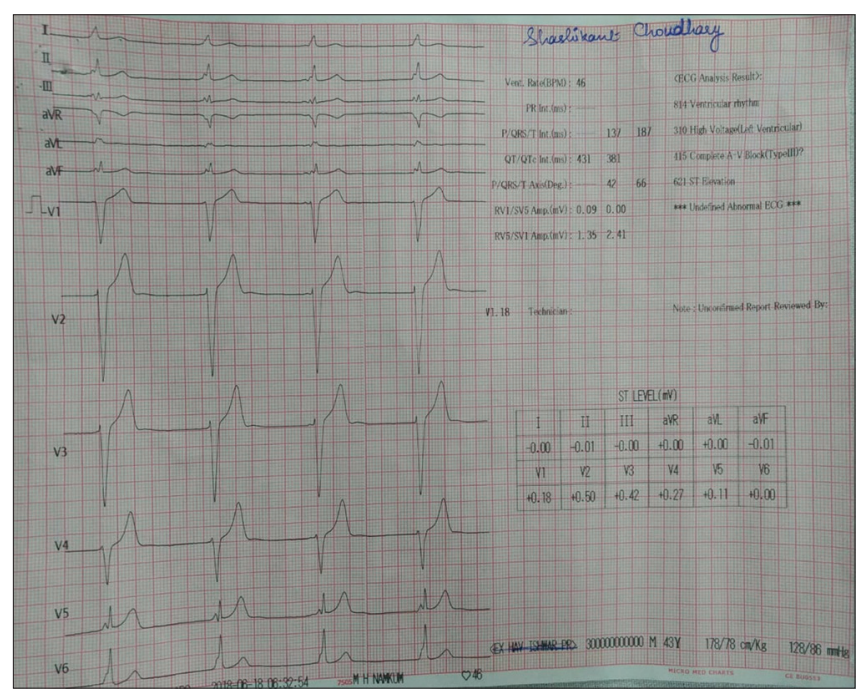

Figure 3: Electrocardiography showing junctional rhythm

was started on specific treatment with antibiotics to which he responded well.

\section{Financial support and sponsorship}

Nil.

Conflicts of interest

There are no conflicts of interest.

\section{ReFERENCES}

1. Sambasiva RR, Naveen G, Bhalla P, Agarwal SK. Leptospirosis in India and the rest of the world. Braz J Infect Dis 2003;7:178-93.

2. Sehgal SC. Epidemiological patterns of leptospirosis. Indian J Med Microbiol 2006;24:310-1.

3. Younes-Ibrahim M, Burth P, Castro-Faria M, Cheval L, Buffin-Meyer B, Marsy S, et al. Effect of leptospira interrogans endotoxin on renal tubular Na, K-ATPase and H, K-ATPase activities. Ann N Y Acad Sci 1997;834:684-6

4. Rajiv C, Manjuran RJ, Sudhayakumar N, Haneef M. Cardiovascular involvement in leptospirosis. Indian Heart J 1996;48:691-4.

5. Trivedi SV, Bhattacharya A, Amichandwala K, Jakkamsetti V. Evaluation of cardiovascular status in severe leptospirosis. J Assoc Physicians India 2003;51:951-3.

6. Navinan MR, Rajapakse S. Cardiac involvement in leptospirosis. Trans R Soc Trop Med Hyg 2012;106:515-20. 